\title{
ON THE VANISHING OF POINCARÉ SERIES OF RATIONAL FUNCTIONS
}

\author{
BY IRWIN KRA ${ }^{1}$
}

1. Let $\Gamma$ be a finitely generated nonelementary Kleinian group with region of discontinuity $\Omega$ and limit set $\Lambda$. Let $\lambda(z)|d z|$ be the Poincaré metric on $\Omega$ (normalized to have constant negative curvature -1 ). Let $q \in \mathbf{Z}, q \geq 2$. A cusp form for $\Gamma$ of weight $(-2 q)$ is a holomorphic function $\varphi$ on $\Omega$ satisfying

$$
\varphi(\gamma z) \gamma^{\prime}(z)^{q}=\varphi(z), \text { for all } \gamma \in \Gamma, \text { for all } z \in \Omega,
$$

and either (hence both) of the following equivalent conditions:

$$
\begin{gathered}
\iint_{\Omega / \Gamma} \lambda(z)^{2-q}|\varphi(z) d z \wedge d \bar{z}|<\infty \\
\sup _{z \in \Omega}\left\{\lambda(z)^{-q}|\varphi(z)|\right\}<\infty .
\end{gathered}
$$

The equivalence of (2) and (3) shows that the Peterson scalar product

$$
\langle\varphi, \psi\rangle=i \iint_{\Omega / \Gamma} \lambda(z)^{2-2 q} \varphi(z) \overline{\psi(z)} d z \wedge \overline{d z}
$$

induces a Hilbert space structure on the space of cusp forms.

Let $\Delta$ be a $\Gamma$-invariant union of components of $\Omega$, and define $\mathbf{A}_{q}(\Delta)$ to be the space of cusp forms for $\Gamma$ of weight $(-2 q)$ that vanish on $\Omega \backslash \Delta$. Abbreviate $\mathbf{A}_{q}(\Omega)$ by $\mathbf{A}_{q}{ }^{2}$

Define $R_{q}$ to be the space of rational functions $f$ such that

(5) $f$ is holomorphic on $\Omega$,

(6) $f$ has only simple poles (on $\Lambda$ ), and

$$
\begin{aligned}
& f(z)=O\left(|z|^{-2 q}\right), \quad z \rightarrow \infty \text { if } \infty \in \Omega, \text { and } \\
& f(z)=O\left(|z|^{-(2 q-1)}\right), \quad z \rightarrow \infty \text { if } \infty \in \Lambda .
\end{aligned}
$$

If $f \in R_{q}$, then the Poincaré series

$$
\sum_{\gamma \in \Gamma} f(\gamma z) \gamma^{\prime}(z)^{q}, \quad z \in \Omega
$$

converges absolutely and uniformly on compact subsets of $\Omega$ and defines a cusp form $\Theta_{q} f \in \mathbf{A}_{q}$. Bers [3] has shown that

Received by the editors August 16, 1982 and, in revised form, September 20, 1982.

1980 Mathematics Subject Classification. Primary 10D15, 30F40.

${ }^{1}$ Research partially supported by NSF grant MCS8102621.

${ }^{2}$ The group $\Gamma$ is fixed throughout this paper. We hence suppress in the notation the dependence on $\Gamma$ of the various spaces and operators considered.

(C) 1983 American Mathematical Society 0273-0979/82/0000-1040/\$02.00 


$$
\Theta_{q}: R_{q} \rightarrow \mathbf{A}_{q}
$$

is a surjective linear operator. The starting point of this investigation was the following theorem that quantitatively strengthens Bers' result.

THEOREM 1. Let $a_{1}, \ldots, a_{2 q-1}$ be $(2 q-1)$ distinct points in $\Lambda$, and let $\gamma_{1}, \ldots, \gamma_{N}$ generate $\Gamma$ (define $\left.\gamma_{0}=I\right)$. Then $\Theta_{q} \mid R_{q}^{0}$ is surjective, where $R_{q}^{0}=$ $\left\{f \in R_{q} \mid f\right.$ is holomorphic except possibly at $\gamma_{j}\left(a_{k}\right), k=1, \ldots, 2 q-1, j=$ $0, \ldots, N\} .^{3}$

In certain cases $\Theta_{q} \mid R_{q}^{0}$ is an isomorphism. Spanning sets for $\Gamma$ Fuchsian were obtained by Hejhal [4]. For $q=2$, and $\Gamma$ Fuchsian, Wolpert [11] obtained bases, as did Kra and Maskit [7] for $\Gamma$ geometrically finite function groups.

2. We turn now to the more interesting vanishing problem raised by Poincaré [10, p. 249] (see also Petersson [9] and Hejhal [4]. Find necessary and sufficient conditions for $\Theta_{q} f$ to vanish identically on $\Omega$ (or $\Delta$ ) for $f \in R_{q}$.

For $\psi \in \mathbf{A}_{q}(\Delta)$, the unique Bers potential $F=F_{\psi}$ for the canonical generalized Beltrami coefficient $\mu=\lambda^{2-2 q} \bar{\psi}$ that vanishes at $a_{k}, k=1, \ldots, 2 q-1$, is given by

(9) $F(z)=\frac{\left(z-a_{1}\right) \cdots\left(z-a_{2 q-1}\right)}{2 \pi i} \iint_{\Omega} \frac{\mu(\zeta) d \zeta \wedge d \bar{\zeta}}{(\zeta-z)(\zeta-a) \cdots\left(\zeta-a_{2 q-1}\right)}, \quad z \in \mathbf{C}$.

For $z \in \Lambda \backslash\left\{a_{1}, \ldots, a_{2 q-1}\right\}$, we have (see Kra [5, Chapter V])

$$
F_{\psi}(z)=\langle\varphi(z, \cdot), \psi\rangle \text {, }
$$

where

$$
\varphi(z, \cdot)=\Theta_{q} f(z, \cdot)
$$

and

$$
f(z, \zeta)=\frac{-1}{2 \pi} \frac{1}{\zeta-z} \prod_{j=1}^{2 q-1} \frac{z-a_{j}}{\zeta-a_{j}} .
$$

Note that for $z \in \Lambda \backslash\left\{a_{1}, \ldots, a_{2 q-1}\right\}, f(z, \cdot) \in R_{q}$. We let

$$
\mathcal{F}_{1-q}(\Delta)=\left\{\text { restrictions to } \Lambda \text { of potentials } F_{\psi} \text { with } \psi \in \mathbf{A}_{q}(\Delta)\right\} \text {. }
$$

As usual $\mathcal{F}_{1-q}=\mathcal{F}_{1-q}(\Omega)$. Observe that $\mathcal{F}_{1-q}(\Delta)$ is a finite-dimensional space of continuous functions on $\Lambda$. Also $\mathcal{F}_{1-q}(\Delta) \subset \mathcal{F}_{1-q}$, for all $\Delta$.

If $f \in R_{q}$, then we can find $m \geq 1$ distinct points $b_{1}, \ldots, b_{m}$ in $\Lambda \backslash\left\{a_{1}, \ldots, a_{2 q-1}\right\}$ and complex numbers $\beta_{1}, \ldots, \beta_{m}$ so that

$$
f(\varsigma)=\sum_{j=1}^{m} \beta_{j} f\left(b_{j}, \varsigma\right), \quad \varsigma \in \mathbf{C} .
$$

The points $b_{1}, \ldots, b_{m}$ and the constants $\beta_{1}, \ldots, \beta_{m}$ are uniquely determined by $f$. We now define a surjective linear map

$$
K: R_{q} \rightarrow \mathcal{F}_{1-q}^{*}
$$

\footnotetext{
${ }^{3}$ If $\gamma_{j}\left(a_{k}\right)=\infty$, then holomorphicity at this point means $f(z)=O\left(|z|^{-2 q}\right), z \rightarrow \infty$. Conventions regarding $\infty$ will henceforth be ignored.
} 
from $R_{q}$ to the dual space of $\mathcal{F}_{1-q}$ by the formula

$$
\mathcal{K}(f)(F)=\sum_{j=1}^{m} \beta_{j} F\left(b_{j}\right), \quad F \in \mathcal{F}_{1-q},
$$

where $f \in R_{q}$ is given by (13).

THEOREM 2. Given $f \in R_{q}$, then

$$
\Theta_{q} f|\Delta=0 \Leftrightarrow \mathcal{K}(f)| \mathcal{F}_{1-q}(\Delta)=0 .
$$

The proof uses the duality given by the Petersson scalar product (4) and the identity (10).

Since $K$ is a very simple operator, Theorem 2 shows that the vanishing problem is completely solved if we can construct a basis for $f_{1-q}(\Delta)$.

3. Let $P H_{\Delta}^{1}\left(\Pi_{2 q-2}\right)$ denote the Eichler cohomology group of $\Delta$-parabolic cohomology classes (see Kra [5, Chapter V]), where $\Pi_{2 q-2}$ is the space of polynomials of degree $\leq 2 q-2$, and let $P H^{1}\left(\Pi_{2 q-2}\right)$ denote the space cohomology classes that are parabolic with respect to all parabolic elements of $\Gamma$. Given $\psi \in \mathbf{A}_{q}(\Delta)$, then

$$
\gamma \mapsto F_{\psi}(\gamma)\left(\gamma^{\prime}\right)^{1-q}-F_{\psi}, \quad \gamma \in \Gamma,
$$

defines a cohomology class $\beta^{*}(\psi) \in P H^{1}\left(\Pi_{2 q-2}\right)$, known as the Bers class of $\psi$.

THEOREM 3. If the Bers map

$$
\beta^{*}: \mathbf{A}_{q} \rightarrow P H^{1}\left(\Pi_{2 q-2}\right)
$$

is surjective, then $F_{1-q}$ can be determined algebraically from the parabolic $\Pi_{2 q-2}$-cocycles for the group $\Gamma$.

We must explain what we mean by determining $f_{1-q}$ algebraically. Let us assume that $a_{1}, \ldots, a_{2 q-1}$ are fixed points of loxodromic elements of $\Gamma$. Theorem 3 means that we can construct algebraically the values at the loxodromic fixed points of functions $F_{1}, \ldots, F_{d}$ that form a basis for $f_{1-q}$. In the proof, we use the fact that if the continuous function $F$ on $\Lambda$ represents the cocycle $\chi$; that is, if

$$
F(\gamma z) \gamma^{\prime}(z)^{1-q}-F(z)=\chi(\gamma)(z), \quad z \in \Lambda,
$$

then for $b \in \Lambda$, a fixed point of a loxodromic element $g \in \Gamma$, we must have

$$
F(b)=\chi(g)(b)\left[g^{\prime}(b)^{1-q}-1\right]^{-1} .
$$

4. The map $\beta^{*}$ of Theorem 3 is surjective for many geometrically finite function groups (Nakada [8]); in particular, for Fuchsian, quasi-Fuchsian, and Schottky groups. In principle, there is an algorithm for each such group to decide when $\Theta_{q} f=0$ for a given $f \in R_{q}$. We state our most explicit construction of such an algorithm in 
THEOREM 4. Let $\Gamma$ be a Schottky group or a finitely generated Fuchsian or quasi-Fuchsian group of the first kind given by a standard presentation on a canonical set of generators. Let $f \in R_{q}$ have poles only at loxodromic fixed points. Then we can write down a (finite) algorithm that determines whether or $\operatorname{not} \Theta_{q} f=0$.

5. Let $\Gamma$ be a finitely generated Fuchsian group of the first kind acting on the unit disk $\Delta$. Then $\Lambda=\partial \Delta=$ the unit circle, and $\Omega=\{z \in \mathbf{C}|| z \mid \neq 1\} \cup\{\infty\}$. To determine when a Poincaré series $\Theta_{q} f, f \in R_{q}$, vanishes identically only on $\Delta$, we need to select $\mathcal{F}_{1-q}(\Delta)$ from $\mathcal{F}_{1-q}$. A not entirely satisfactory answer is contained in

THEOREM 5. Let $\Gamma$ be a finitely generated Fuchsian group of the first kind acting on the unit disk $\Delta$. Then there exists an integer $n=n(q)$ such that for $F \in \mathcal{F}_{1-q}$, we have

$$
F \in \mathcal{F}_{1-q}(\Delta) \Leftrightarrow \int_{0}^{2 \pi} e^{i(1-k-2 q) \theta} F\left(e^{i \theta}\right) d \theta=0 \quad \text { for } k=0,1, \ldots, n .
$$

The debt of this paper to the fundamental contributions of Ahlfors [1] and Bers [2] is obvious, and I am delighted to acknowledge it. Hejhal's paper [4], which contains a somewhat less explicit solution to the vanishing problem for a more limited class of groups, was a useful reminder that this problem should have an algebraic solution. Our solution differs radically from Hejhal's. We rely in very basic ways on the Eichler cohomology machinery $[\mathbf{1}, \mathbf{2}, \mathbf{5}]$. I am happy to thank M. Sheingorn for his insistence that the vanishing problem is important and interesting. Complete proofs and applications will appear elsewhere [6].

\section{REFERENCES}

1. L. V. Ahlfors, Finitely generated Kleinian groups, Amer. J. Math. 86 (1964), 413-429; 87 (1965), 759.

2. L. Bers, Inequalities for finitely generated Kleinian groups, J. Analyse Math. 18 (1967), 23-41.

3. __ Poincaré series for Kleinian groups, Comm. Pure Appl. Math. 26 (1973), 667-672; 27 (1974), 583.

4. D. A. Hejhal, Monodromy groups and Poincaré series, Bull. Amer. Math. Soc. 84 (1978), 339-376.

5. I. Kra, Automorphic forms and Kleinian groups, Benjamin, Reading, Mass., 1972.

6. __ On the vanishing of and spanning sets for Poincaré series for cusp forms (to appear).

7. I. Kra and B. Maskit, Bases for quadratic differentials, Comment. Math. Helv. (to appear).

8. M. Nakada, Quasi-conformal stability of finitely generated function groups, Tôhoku Math. J. 30 (1978), 45-58.

9. H. Petersson, Die linearen Relationen zwischen den ganzen Poincaréschen Reihen von reeller Dimension zur Modulgruppe, Abh. Math. Sem. Hamb. Univ. 12 (1938), 415-472.

10. H. Poincaré, Memoire sur les fonctions fuchsiennes, Acta Math. 1 (1882), 193-294.

11. S. Wolpert, The Fenchel-Nielsen deformation, Ann. of Math. (2) 115 (1982), 501-528.

DEPARTMENT OF MATHEMATICS, STATE UNIVERSity OF NEW YORK AT STONY BROOK, STONY BROOK, NEW YORK 11794 